\title{
Salvage high-dose chemotherapy for children with extragonadal germ-cell tumours
}

\section{U De Giorgi, ${ }^{*}$, G Rosti', S Slavin ${ }^{2}$, I Yaniv ${ }^{3}$, JL Harousseau ${ }^{4}$, R Ladenstein ${ }^{5}$, T Demirer ${ }^{6}$ and G Dini ${ }^{7}$, on behalf of the European Group for Blood and Marrow Transplantation Solid Tumours and Paediatric Disease Working Parties}

IIstituto Oncologico Romagnolo-Department of Oncology/Haematology, Santa Maria delle Croci Hospital, Ravenna, Italy; ${ }^{2}$ Department of Bone Marrow Transplantation and Cancer Immunotherapy, Hadassah University Hospital, Jerusalem, Israel; ${ }^{3}$ Department of Paediatric Haematology Oncology, Schneider Children's Medical Centre of Israel, Petah Tikva, Israel; ${ }^{4}$ Department of Haematology, University Hospital, Nantes, France; ${ }^{5}$ Department of Paediatric Haemato-Oncology, St Anna Children's Hospital, Vienna, Austria; ${ }^{6}$ Department of Haematology/Oncology and Bone Marrow Transplant Unit Ankara University Medical School, Ankara, Turkey; ${ }^{7}$ Department of Paediatric Haematology Oncology, Institute G Gaslini, Genoa, Italy

We reviewed the European Group for Blood and Marrow Transplantation (EBMT) experience with salvage high-dose chemotherapy (HDC) in paediatric patients with extragonadal germ-cell tumour (GCT). A total of 23 children with extragonadal GCT, median age 12 years (range I -20), were treated with salvage HDC with haematopoietic progenitor cell support. The GCT primary location was intracranial site in nine cases, sacrococcyx in eight, retroperitoneum in four, and mediastinum in two. In all, 22 patients had a nongerminomatous GCT and one germinoma. Nine patients received HDC in first- and 14 in second- or third-relapse situation. No toxic deaths occurred. Overall, 16 of 23 patients (70\%) achieved a complete remission. With a median follow-up of 66 months (range $31-173$ months), 10 (43\%) are continuously disease-free. Of six patients who had a disease recurrence after HDC, one achieved a disease-free status with surgical resection followed by chemotherapy and radiotherapy. In total, II patients (48\%) are currently disease-free. Eight of I 4 patients (57\%) with extracranial primary and three of nine patients (33\%) with intracranial primary GCT are currently disease-free. HDC induced impressive long-term remissions as salvage treatment in children with extragonadal extracranial GCTs. Salvage HDC should be investigated in prospective trials in these patients.

British Journal of Cancer (2005) 93, 4I2-417. doi:I0.1038/sj.bjc.6602724 www.bjcancer.com

Published online 2 August 2005

(c) 2005 Cancer Research UK

Keywords: extragonadal germ cell tumour; high-dose chemotherapy; salvage therapy; children

Germ-cell tumours (GCTs) represent 3\% of all paediatric neoplasms (Ries et al, 2003). The most common primary sites are the ovary $(26 \%)$, the coccyx $(24 \%)$, the testis $(18 \%)$, the brain $(18 \%)$, the mediastinum $(4 \%)$ and the retroperitoneum $(4 \%)$. Therefore, germ cell malignancies arising from extragonadal primary site represent nearly $50-60 \%$ of all GCTs in children (Gobel et al, 2000). Extragonadal primary location coupled with high serum levels of alpha-fetoprotein (AFP) (more than $10000 \mathrm{ng} \mathrm{ml}^{-1}$ ) at diagnosis are the most unfavourable prognostic factors for these children (Mann et al, 1989; Marina et al, 1992; Baranzelli et al, 1999, 2000; Schneider et al, 2000; Lo Curto et al, 2003). Moreover, the prognosis of extragonadal GCT is significantly influenced by the histological differentiation, with the secreting GCTs (embryonal carcinoma, yolk sac tumour, choriocarcinoma) and immature teratoma having a higher risk of relapse after primary treatment than germinoma/dysgerminoma and mature teratoma (Gobel et al, 1999).

Conventional treatment for children with advanced GCT consists of cisplatin-based chemotherapy followed by surgical resection of residual disease, if necessary (Rescorla and Breitfeld,

\footnotetext{
* Correspondence: Dr U De Giorgi. Current address: Istituto Toscano Tumori-Department of Oncology, San Giuseppe Hospital, Via Paladini 40, 50053 Empoli (Florence), Italy; E-mail: ugo_degiorgi@yahoo.com Received 9 May 2005; revised 27 June 2005; accepted 28 June 2005; published online 2 August 2005
}

1999). More than $80 \%$ of these children are cured with standard treatments (Mann et al, 2000; Kramarova et al, 2001). Very limited data are available concerning therapeutic options and long-term results of recurrent GCTs in children (Gobel et al, 2002).

High-dose chemotherapy (HDC) with haematopoietic progenitor cell support (HPCS) is a therapeutic option mainly investigated in adult patients with GCT either as first-line or salvage setting (De Giorgi et al, 2002). Preliminary experiences with HDC in children resulted in long-term remissions in those patients in whom a clinical complete remission (CR) could be achieved prior to HDC (Gobel et al, 2002). Subsequently, in children, HDC with HPCS has been mainly investigated as first-line treatment, or for consolidation therapy after primary standard-dose chemotherapy.

To better characterise the role of HDC with HPCS as salvage therapy for children with extragonadal GCT, the large database of the patients registered with the European Group for Blood and Marrow Transplantation (EBMT) was reviewed. This report describes the EBMT experience of salvage HDC in 23 children with extragonadal GCT.

\section{MATERIALS AND METHODS}

\section{Data collection}

The 23 relapse children analysed in this study belong to a cohort of 160 paediatric and adult patients with a diagnosis of extragonadal 
GCT, who have been registered with the EBMT from December 1987 to December 1999. The date of last follow-up was July 2003. Germ-cell tumours were classified as seminoma/germinoma, embryonal carcinoma, choriocarcinoma or mixed GCTs, mature or immature teratoma and yolk sac tumour according to the World Health Organization classification. Patients with histologically undifferentiated tumours with markedly elevated serum markers, who were treated according to GCT protocols, are included in this report. We reviewed the registration details of these patients. The reporting physicians were contacted and asked to provide further information on primary tumour site and extent of disease, histology, tumour markers, initial treatment, second-line chemotherapy, HDC drugs and toxicities, follow-up and data on possible secondary neoplasm. For data collection, a standardised questionnaire was sent to each centre. All patient data were obtained in an anonymous manner. Of 160 registered cases, 120 (75\%) questionnaires were returned. Among these 120 patients, we analysed 23 cases of children with a diagnosis of extragonadal GCTs treated with salvage HDC, including three of nine cases with intracranial GCT, aged 15, 18 and 19 years, respectively. As this is a report of registry data, there are cases where information is incomplete, as indicated in the tables.

\section{Definitions}

Tumour response was classified as follows: CR was defined as a complete disappearance of all clinical, radiological and biochemical evidence of disease, with normalisation of the tumour markers, beta-human chorionic gonadotropin (HCG) and/or AFP and/or lactate dehydrogenase (LDH), for at least a 1-month duration. A partial response (PR) was defined as a decrease in $50 \%$ or more of the sum of the products of perpendicular diameters of measurable disease, lasting at least for 1 month. If elevated markers were the only evidence of disease, a decrease of $90 \%$ or greater was required for a PR. In addition, reduction of the size of a tumour lesion and normalisation of previously elevated tumour markers was considered a partial remission with tumour marker normalisation $(\mathrm{PR}-)$, whereas a reduction $\geqslant 50 \%$ in the sum of the perpendicular diameters of measurable disease plus a tumour marker decrease for at least 1 month, but without complete normalisation, was considered a marker positive partial remission $(\mathrm{PR}+)$. Stable disease (SD) was defined as a decrease $<50 \%$ or an increase $<25 \%$ in bidimensional tumour measurements or stable tumour marker levels. Progressive disease (PD) was defined as either residual lesions increasing in size or as the occurrence of new lesions and/or elevation of tumour markers at repeated controls.

\section{Patient characteristics}

Details at diagnosis of the 23 patients are listed in Table 1. The median age was 7 years (range, 1-19). In all, 14 patients were males and nine were female. Nine patients had primary CNS (pineal/pituitary area), eight sacrococcygeal, four retroperitoneal and two mediastinal GCT. A total of 22 patients had a nongerminoma (three embryonal carcinoma, three yolk sac tumour, two immature teratoma, one choriocarcinoma, four CNS lesions with serum tumour marker increase, nine mixed GCT) and one germinoma. Most commonly, patients received cisplatin-based chemotherapy as first-line treatment. Table 1 summarises the characteristics of patients at diagnosis, primary treatment and response.

\section{Salvage treatment}

In all, 13 patients received a second-line standard-dose chemotherapy given at first relapse/progression, while one patient received even a third-line standard-dose chemotherapy at second relapse/progression. Nine patients received salvage HDC in first-, 13 in second- and one in third-relapse situation. Nine (39\%) patients received an induction and/or mobilising regimen before

Table I Patient characteristics at diagnosis and treatments before high-dose chemotherapy

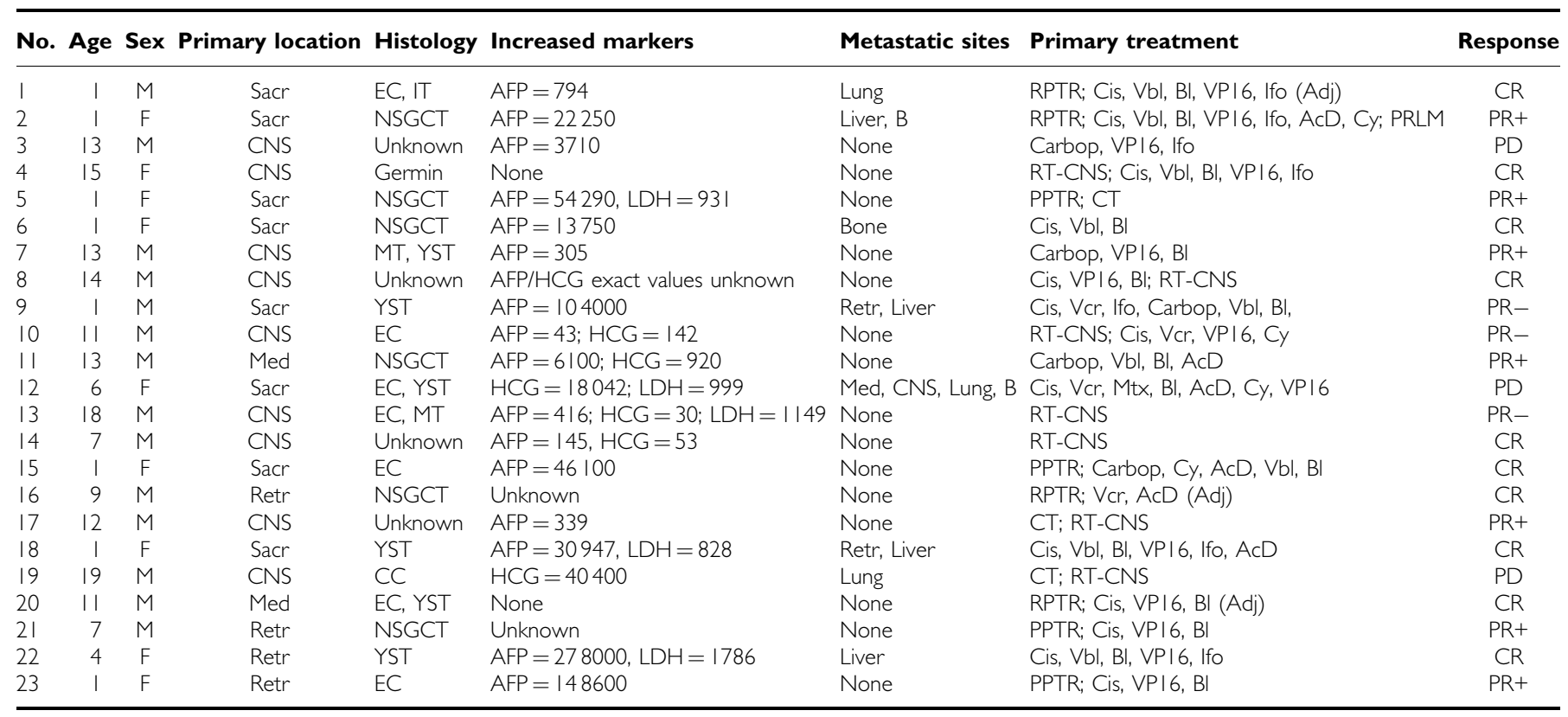

M, male; F, female; Sacr, sacrococcix; CNS, central nervous system; Med, mediastinum; Retr, retroperitoneum; B, bone; IT, immature teratoma; MT, mature teratoma; YST, yolk sac tumour; EC, embryonal carcinoma; CC, choriocarcinoma; Germin, germinoma; NSGCT, nonseminomatous germ-cell tumour (not furthermore specified); AFP, alphafetoprotein $\left(\mathrm{ng} \mathrm{ml}^{-1}\right)$; HCG, human choriogonadotropin $\left(\mathrm{IU} \mathrm{I}^{-1}\right)$; RPTR, radical primary tumour resection; PPTR, partial primary tumour resection; PRLM, partial resection liver metastases; Cis, cisplatin; Vbl, vinblastin; Bl, bleomycin; VPI6, etoposide; Ifo, ifosfamide; AcD, actinomycin D; Cy, cyclophosphamide; Carbop, carboplatin; Vcr, vincristine; Mtx, methotrexate; $\mathrm{CT}$, chemotherapy (schedule/drugs not available); RT, radiotherapy; Adj, adjuvant; CR, complete remission; PR-, marker-negative partial remission; PR+, markerpositive partial remission; SD, stable disease; PD, progressive disease. 
Table 2 High-dose chemotherapy regimens

\begin{tabular}{|c|c|c|}
\hline Abbreviation & Chemotherapeutic drugs & $\begin{array}{l}\text { No. HDC } \\
\text { courses }\end{array}$ \\
\hline CarboPEC & $\begin{array}{l}\text { Carboplatin } 250-350 \mathrm{mg} \mathrm{m}^{-2} \times 4 \text { days or dosed with Calvert formula with } A \cup C=7 \text { (lower dosed used) } \\
\text { Etoposide } 250-400 \mathrm{mg} \mathrm{m}^{-2} \times 4 \text { days } \\
\text { Cyclophosphamide } 1.6 \mathrm{~g} \mathrm{~m}^{-2} \times 4 \text { days }\end{array}$ & 7 \\
\hline CE & $\begin{array}{l}\text { Carboplatin } 250-500 \mathrm{mg} \mathrm{m}^{-2} \times 3-4 \text { days or dosed with Calvert formula with } A \cup C=7 \text { (lower dosed used) } \\
\text { Etoposide } 250-400 \mathrm{mg} \mathrm{m}^{-2} \times 3-4 \text { days }\end{array}$ & 5 \\
\hline TE & $\begin{array}{l}\text { Thiotepa } 300 \mathrm{mg} \mathrm{m}^{-2} \times 3 \text { days } \\
\text { Etoposide } 250-300 \mathrm{mg} \mathrm{m}^{-2} \times 3 \text { days }\end{array}$ & 5 \\
\hline CarboPETM & $\begin{array}{l}\text { Carboplatin } 250-350 \mathrm{mg} \mathrm{m}^{-2} \times 3-4 \text { days or dosed with Calvert formula with } A \cup C=7 \text { (lower dosed used) } \\
\text { Etoposide } 250-400 \mathrm{mg} \mathrm{m}^{-2} \times 3-4 \text { days } \\
\text { Thiotepa } 200-250 \mathrm{mg} \mathrm{m}^{-2} \times 2-3 \text { days } \\
\text { Melphalan } 80-100 \mathrm{mg} \mathrm{m}^{-2} \times 1 \text { day }\end{array}$ & 2 \\
\hline Other regimens & $\begin{array}{l}\text { HD-CyC = high-dose cyclophosphamide } 7 \mathrm{~g} \mathrm{~m}^{-2}(n=2) ; \mathrm{CM}=\text { cyclophosphamide/melphalan }(n=2) ; \mathrm{TM}=\text { thiotepa/melphalan } \\
(n=1) ; \mathrm{TC}=\text { thiotepa/cyclophasphamide }(n=\mathrm{I}) ; \mathrm{ICE}=\text { ifosfamide/carboplatin/etoposide }(n=1) ; \text { HD-PEC = cisplatin/etoposide/ } \\
\text { cyclophosphamide }(n=1) ; \text { CarboPTC = carboplatin/thiotepa/cyclophosphamide }(n=1) ; \text { not available }(n=1)\end{array}$ & \\
\hline
\end{tabular}

late-intensification HDC. The response to the last chemotherapeutic regimen before the induction regimen was CR in four cases, $\mathrm{PR}-$ in two and PR + in three (Table 3 ). Then, the policy of EBMT centres was to use late-intensification HDC only in chemosensitive disease. In total, $14(61 \%)$ patients were treated with upfront salvage HDC. The response to the last chemotherapeutic regimen before upfront HDC was PR + in three cases, SD in one, PD in three, while six patients underwent HDC at the time of relapse after a previous $\mathrm{CR}$ or $\mathrm{PR}-$ (sensitive relapse) (Table 3 ). The salvage HDC regimens for extragonadal GCT patients were adapted based on the chemotherapeutic regimens given as initial therapy, and the salvage HDC protocols used in each centre for recurrent tumours in children, including drugs proven to be active in GCT. A total of 18 patients received one course of HDC, four underwent two courses; in one case three courses were given. The most commonly used HDC regimen was Carbopec, including carboplatin, etoposide and cyclophosphamide $(n=7,24 \%)$. High-dose chemotherapy regimens are listed in Table 2. Haematopoietic support consisted of peripheral blood progenitor cells (PBPCs) in 19 courses, autologous bone marrow transplantation (ABMT) in nine, both of them in one. Table 3 summarises the salvage HDC treatments and results.

\section{Statistical analysis}

Descriptive statistics are presented as the median and range. Duration of followup and survival in this analysis were calculated based on the date of the first day of salvage chemotherapy until the date of last contact, if the patient was still alive, or until the date of death. Probabilities of disease-free survival and overall survival were determined using the Kaplan-Meier product limit method (Kaplan and Meier, 1958).

\section{RESULTS}

\section{Toxicity}

Toxicity data were fully assessable for 26 (90\%) of 29 HDC cycles delivered. No treatment-related deaths occurred after HDC.

The median time to recovery of an absolute neutrophil count $>500 \mu \mathrm{l}^{-1}$ and a platelet count $>20000 \mu \mathrm{l}^{-1}$, respectively, was 10.5 days (range, $6-22$ ) and 10.5 days (range, $0-48$ ). The median number of transfusions of red blood cell and platelet bags was 4 (range, 1-8) and 6.5 (range, 1-30), respectively. Fever occurred in 21 courses $(81 \%)$, with an overall median duration of 3 days (range, $0-13$ ). The number of HDC courses with episodes of clinically documented infections was 13 (50\%).

The following nonhaematological side effects were the most relevant: grade $\geqslant 3$ stomatitis was reported in nine courses, grade $\geqslant 3$ pulmonary toxicity in two, grade 3 peripheral neurotoxicity in one, grade 3 anorexia in one and psychosis in one. Veno-occlusive disease occurred in two patients (pt. no. 8 and no. 16, Table 3), both received the HDC regimen, including carboplatin, etoposide, thiotepa and melphalan. Neither renal nor cardiac toxicity was observed. No patients developed myelodysplasia or secondary neoplasms after HDC.

\section{Response and survival}

Overall, $16(70 \%)$ patients achieved a CR. Of these patients, 14 obtained a radiological CR after HDC, while the other two with radiological $\mathrm{PR}-$ obtained a $\mathrm{CR}$ with post-HDC resection of residual masses and radiotherapy in one case. Results are presented in detail in Table 3.

The median follow-up period for all patients was 31 months (range, 3-173 months) and 66 months (range, 31-173) for surviving patients. Of 23 patients, 10 (43\%) are continuously disease-free. Of six patients who had a disease recurrence after HDC, one underwent further surgery, chemotherapy and radiotherapy, and achieved a disease-free status. In total, 11 patients $(48 \%)$ are currently disease-free (Figure 1$)$.

The continuously disease-free status was achieved in four of nine patients $(44 \%)$ with chemosensitive disease treated with lateintensification HDC, in four of six patients (67\%) with sensitive relapse, who underwent upfront HDC, and in two of eight patients $(25 \%)$ with $\mathrm{PR}+$ or SD, or PD, receiving upfront HDC (Table 3 ).

Eight of 14 patients (57\%) with extracranial primary GCT (five of eight patients $(62 \%)$ with sacrococcygeal, two of four patients $(50 \%)$ with retroperitoneal and one of two patients $(50 \%)$ with mediastinal primary GCT) and three of nine patients (33\%) with intracranial primary GCT are currently alive disease-free.

Figure 2 illustrates the outcome of patients with extragonadal GCT, according to the time of relapse. The 2-year overall survival rates for first relapsing patients and second-third relapsing patients were 78 and $43 \%$, respectively. 
Table 3 Salvage high-dose chemotherapy for children with extragonadal germ-cell tumour

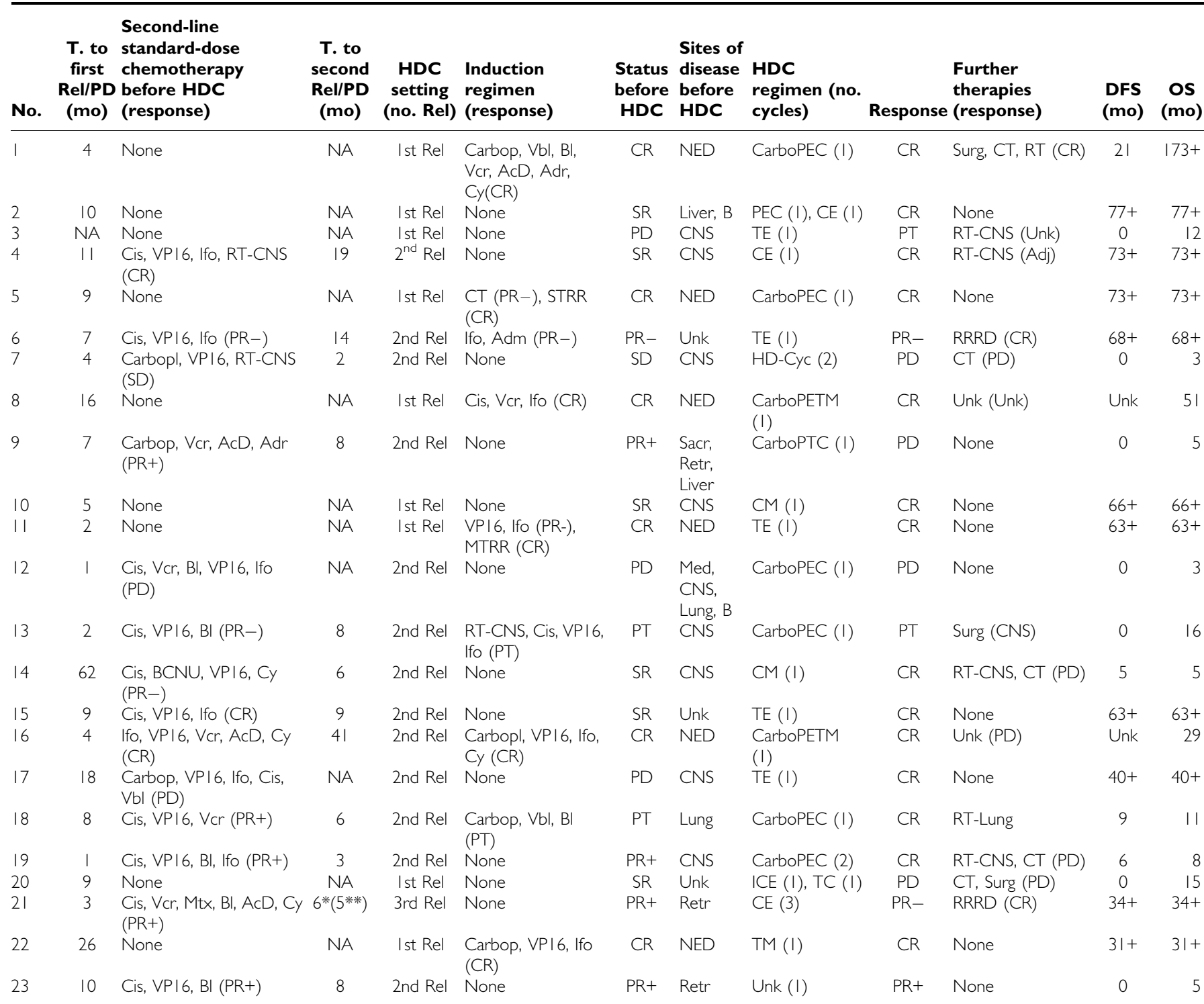

*After 6 months second Rel, treated with third-line standard-dose chemotherapy with Ifo,Vbl, VPI 6 (PR+); **after 5 months 3rd Rel/PD. Abbreviations: T.to first or second Rel/ PD, Time to first or second relapse/progressive disease; HDC, high-dose chemotherapy; mo, months; NA, not applicable; Sacr, sacrococcix; CNS, central nervous system; Retr, retroperitoneum; Med, mediastinum; B, bone; NED, not evidence of disease; Unk, unknown; IT, immature teratoma; MT, mature teratoma; YST, yolk sac tumour; EC, embryonal carcinoma; Germ, germinoma; CC, choriocarcinoma; AFP, alpha-fetoprotein $\left(\mathrm{ng} \mathrm{m}^{-1}\right)$; HCG, human choriogonadotropin (IUI $\mathrm{I}^{-1}$ ); MTRR, mediastinal tumour radical resection; STRR, sacrococcigeal tumour radical resection; RRRD, radical resection of residual disease; Cis, cisplatin; Vbl, vinblastin; Bl, bleomycin; VPI6, etoposide; Ifo, ifosfamide; ActD, actinomycin D; Cy, cyclophosphamide; Carbop, carboplatin; Vcr, vincristine; BCNU, bendamustine; Mtx, methotrexate; Adm, adriamycin; CT, chemotherapy (schedule/drugs not available); RT, radiotherapy; Adj, adjuvant; CR, complete remission; PR-, marker-negative partial remission; PR+, marker-positive partial remission; SD, stable disease; PD, progressive disease; PT, persistent tumour with response not evaluable; SR, sensitive relapse (indicating patients relapsing after a previous CR or PR- and receiving frontline HDC without induction regimens). For abbreviations of HDC regimens, see Table 2.

\section{DISCUSSION}

Children with relapsing/progressing extragonadal GCT are characterised by a poor prognosis, with a salvage rate in the first relapse in the range of $40-45 \%$ (Marina et al, 1992; Baranzelli et al, 1999; Sawamura et al, 1999; Gobel et al, 2000; Mann et al, 2000; Schmugge et al, 2000).

According to extragonadal primary location, histology and the site of recurrence/progression, the salvage strategies are different. Standard-dose cisplatin- or carboplatin-based chemotherapy is the conventional treatment for most of these patients. In patients with local recurrence of sacrococcygeal GCT, the surgical complete resection represents the cornerstone of the salvage treatment, while chemotherapy is employed in recurrent inoperable and metastatic disease (Schneider et al, 2001). In patients with recurrent CNS GCTs, chemotherapy alone or combined with radiotherapy has to be considered, if feasible (Sawamura et al, 1999; Kellie et al, 2004).

In children with recurrent mediastinal or retroperitoneal primary GCT, the salvage strategy consists of chemotherapy followed by resection of residual masses, if necessary (Gobel et al, 2000). High-dose chemotherapy is currently investigated as a possible option for high-risk and recurrent CNS GCTs, and is used on an individual basis as salvage therapy for children with other extragonadal germ-cell malignancies (Calaminus and Patte, 2002; Cushing et al, 2004; Kellie et al, 2004; Modak et al, 2004). 


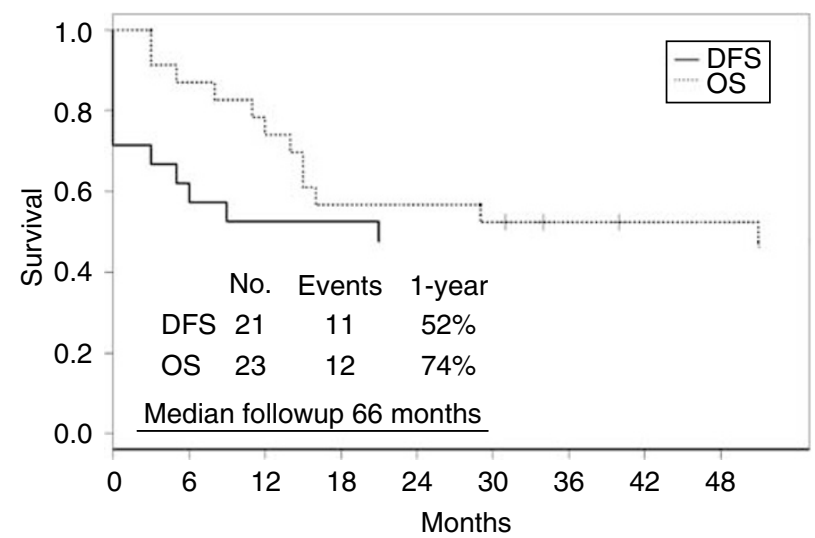

Figure I Disease-free and overall survival for patients with relapsing extragonadal GCT. Note: 21 of all 23 patients are assessable for diseasefree survival (see Table 3).

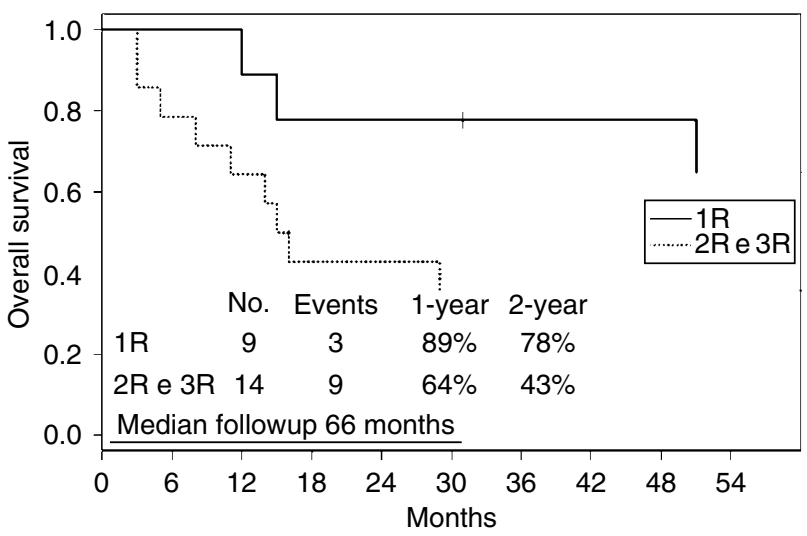

Figure 2 Overall survival with respect to the first (nine patients) and further relapses ( 14 patients).

Few series with salvage chemotherapy for relapse GCT patients with extragonadal primary are reported in literature. The largest experience with different salvage standard-dose chemotherapeutic approaches, more often including cisplatin-based chemotherapy, in relapse patients with sacrococcygeal GCT showed 10 of the 22 patients $(45 \%)$ disease-free at a median followup of 56 months (Schneider et al, 2001). Other authors investigated the use of salvage treatments including standard-dose chemotherapy and/or HDC in patients with relapsed CNS GCT (Calaminus and Patte, 2002; Kellie et al, 2004). Overall, in these highly heterogeneous experiences, long-term remissions were obtained in nearly 25$50 \%$ of cases. Another report presented the largest series of patients with recurrent or progressive CNS GCTs treated with thiotepa-based HDC. No toxic deaths occurred. Out of the 21 patients, 11 (52\%) achieved long-term disease-free survival. Results were clearly better for germinomas (seven of nine patients, $78 \%$, disease-free continuously), than for nongerminomatous GCTs (four of 12 patients, 33\%, long-term disease-free) (Modak et al, 2004). Recently, a regimen including high doses of cisplatin has been compared to standard-dose chemotherapy as primary treatment in a randomised study in 299 children with high-risk GCTs, including 165 extragonadal GCTs. Chemotherapy has consisted of bleomycin $15 \mathrm{U} \mathrm{m}^{-2}$, etoposide $500 \mathrm{mg} \mathrm{m}^{-2}$ and either cisplatin $200 \mathrm{mg} \mathrm{m}^{-2}$ (high-dose arm) or cisplatin $100 \mathrm{mg} \mathrm{m}^{-2}$ (standard-dose arm). The overall survival has been similar in both regimens; treatment-related deaths and grade 3-4 toxicities have been more common with HDC. As a consequence, the arm including high-dose cisplatin is not being recommend for paediatric patients as first-line treatment (Cushing et al, 2004).

In this report, we have presented the results of the EBMT experience with HDC as salvage treatment for children with extragonadal GCT. To the best of our knowledge, this is the largest reported experience with HDC in these patients as salvage setting. Only one patient with germinoma (pt. no. 4 in the tables) was included in this analysis. Nine patients received HDC in first- and 14 in second- or third-relapse situation. All extracranial GCT patients but one were chemosensitive before HDC, while two intracranial GCT patients were chemorefractory. With a median followup of 66 months (range, 31-173), of 23 extragonadal GCT patients who received salvage HDC, $10(43 \%)$ are disease-free continuously. Since another patient with disease recurrence achieved a disease-free status after further chemotherapy, 11 extragonadal GCT patients (48\%) are currently disease-free: eight of 14 patients $(57 \%)$ with extracranial primary GCT, and three of nine patients $(33 \%)$ with intracranial primary GCT. Therefore, HDC could be a possible option as salvage treatment for extracranial GCTs. Our results for intracranial GCTs, including only one germinoma, are similar to those of other major experiences for nongerminomatous CNS GCTs (Modak et al, 2004). The 2-year overall survival rates for first relapsing patients and second-third relapsing extragonadal GCT patients were clearly different: 78 and $43 \%$, respectively. In our experience, HDC induces a high rate of long-term remissions even as third-line treatment. An induction regimen before HDC was given in nine patients, five $(56 \%)$ are continuously disease-free. Multiple HDC courses were given in five cases as upfront salvage treatment, in four with PBPC support. Of these five children, three died of disease. Therefore, either an induction regimen before HDC or multiple upfront HDC courses were not associated with improved results (Table 3 ).

In the EBMT experience, no toxic deaths occurred, and the two cases of VOD were reported in the only two patients treated with the four-drug HDC regimen, including carboplatin, etoposide, thiotepa and melphalan. The tolerability of HDC could be related to the HDC regimens used. Most commonly, patients received carboplatin- or etoposide-based HDC regimens, and only in one case high-dose cisplatin was given (Table 2). Moreover, no patients developed myelodysplasia or secondary neoplasms after receiving HDC in our short experience, even though an increased risk of acute myelogenous leukaemia was reported in children with GCT after standard-dose chemotherapy (Schneider et al, 1999).

In conclusion, in the EBMT experience, HDC with HPCS induced impressive long-term remissions as salvage treatment in children with extragonadal extracranial GCTs. Salvage HDC should be investigated in prospective trials in these patients. New strategies should be considered for salvage treatment of patients with CNS GCT.

\section{REFERENCES}

Baranzelli MC, Bouffet E, Quintana E, Portas M, Thyss A, Patte C (2000) Non-seminomatous ovarian germ cell tumours in children. Eur J Cancer 36(3): $376-383$
Baranzelli MC, Kramar A, Bouffet E, Quintana E, Rubie H, Edan C, Patte C (1999) Prognostic factors in children with localized malignant nonseminomatous germ cell tumors. J Clin Oncol 17(4): 1212-1218 
Calaminus G, Patte C (2002) Experience within the SIOP CNS GCT 96 trial/ SFOP studies with HDC/SCR in patients with CNS germ cell tumours and further implications for high risk patients. Bone Marrow Transplant 30(Suppl 1): S34 (abstr. 54)

Cushing B, Giller R, Cullen JW, Marina NM, Lauer SJ, Olson TA, Rogers PC, Colombani P, Rescorla F, Billmire DF, Vinocur CD, Hawkins EP, Davis MM, Perlman EJ, London WB, Castleberry RP (2004) Randomized comparison of combination chemotherapy with etoposide, bleomycin, and either high-dose or standard-dose cisplatin in children and adolescents with high-risk malignant germ cell tumors: a pediatric intergroup study-Pediatric Oncology Group 9049 and Children's Cancer Group 8882. J Clin Oncol 22(13): 2691 - 2700

De Giorgi U, Rosti G, Papiani G, Marangolo M (2002) The status of highdose chemotherapy with hematopoietic stem cell transplantation in germ cell tumor patients. Haematologica 87(1): 95-104

Gobel U, Calaminus G, Schneider DT, Schmidt P, Haas RJ (2002) Management of germ cell tumors in children: approaches to cure. Onkologie 25(1): 14-22

Gobel U, Haas RJ, Jurgens H (1999) Malignant non-testicular germ cell tumors. Monogr Paediatr 18: $327-341$

Gobel U, Schneider DT, Calaminus G, Haas RJ, Schmidt P, Harms D (2000) Germ cell tumors in childhood and adolescence. Ann Oncol 11(3): 263-271

Kaplan EL, Meier P (1958) Nonparametric estimation from incomplete observations. J Am Stat Assoc 53: 457-481

Kellie SJ, Boyce H, Dunkel IJ, Diez B, Rosenblum M, Brualdi L, Finlay JL (2004) Primary chemotherapy for intracranial nongerminomatous germ cell tumors: results of the second international CNS germ cell study group protocol. J Clin Oncol 22(5): 846-853

Kramarova E, Mann JR, Magnani C, Corraziari I, Berrino F (2001) Survival of children with malignant germ cell, trophoblastic and other gonadal tumours in Europe. Eur J Cancer 37(6): 750 - 759

Lo Curto M, Lumia F, Alaggio R, Cecchetto G, Almasio P, Indolfi P, Siracusa F, Bagnulo S, De Bernardi B, De Laurentis T, Di Cataldo A, Tamaro P (2003) Malignant germ cell tumors in childhood: results of the first Italian Cooperative Study 'TCG 91'. Med Pediatr Oncol 41(5): $417-425$

Mann JR, Pearson D, Barrett A, Raafat F, Barnes JM, Wallendszus KR (1989) Results of the United Kingdom children's cancer study of

\section{Appendix}

\section{The following institutions contributed to the study}

S Slavin, D Engelhard Department of Bone Marrow Transplantation and Cancer Immunotherapy, Hadassah University Hospital, Jerusalem, Israel; I Yaniv, S Stein, Department of Paediatric Haematology Oncology, Schneider Children's Medical Center of Israel, Petah Tikva, Israel; JL Harousseau, Department of Haematology, University Hospital, Nantes, France; A Tobler, K Leibundgut, M Fey, Division of Haematology/Oncology, University Hospital, Bern, Switzerland; D Bron, Experimental Haematology, Institut Jules Bordet, Brussels, Belgium; V Castel, Department of Paediatric Oncology, Hospital Infantil La Fe, Valencia, Spain; J Cornish, Department of Paediatric Oncology/BMT, Royal Hospital for Children, Bristol, UK; H Gadner, R Ladenstein, Department of prognostic factors in nonseminomatous germ cell studies. Cancer 63(9): $1657-1667$

Mann JR, Raafat F, Robinson K, Imeson J, Gornall P, Sokal M, Gray E, McKeever P, Hale J, Bailey S, Oakhill A (2000) The United Kingdom Children's Cancer Study Group's second germ cell tumor study: carboplatin, etoposide, and bleomycin are effective treatment for children with malignant extracranial germ cell tumors, with acceptable toxicity. J Clin Oncol 18(22): 3809-3818

Marina N, Fontanesi J, Kun L, Rao B, Jenkins JJ, Thompson EI, Etcubanas E (1992) Treatment of childhood germ cell tumors, review of the St Jude experience from 1979 to 1988. Cancer 70(10): 2568-2574

Modak S, Gardner S, Dunkel IJ, Balmaceda C, Rosenblum MK, Miller DC, Halpern S, Finlay JL (2004) Thiotepa-based high-dose chemotherapy with autologous stem-cell rescue in patients with recurrent or progressive CNS germ cell tumors. J Clin Oncol 22(10): 1934-1943

Rescorla FJ, Breitfeld PP (1999) Pediatric germ cell tumors. Curr Probl Cancer 23(6): 257-303

Ries LAG, Eisner MP, Kosary CL, Hankey BF, Miller BA, Clegg L, Mariotto A, Fay MP, Feuer EJ, Edwards BK (eds) (2003) SEER Cancer Statistics Review, 1975-2000. Bethesda, MD: National Cancer Institute http:// seer.cancer.gov/csr/1975_2000/ (accessed April 2005)

Sawamura Y, Ikeda JL, Tada M, Shirato H (1999) Salvage therapy for recurrent germinomas in the central nervous system. Br J Neurosurg 13(4): $376-381$

Schmugge M, Boltshauser E, Pluss HJ, Niggli FK (2000) Long-term followup and residual sequalae after treatment for intracerebral germ-cell tumour in children and adolescents. Ann Oncol 11(5): 527-533

Schneider DT, Calaminus G, Reinhard H, Gutjahr P, Kremens B, Harms D, Gobel U (2000) Primary mediastinal germ cell tumors in children and adolescents: results of the German Cooperative Protocols MAKEI 83/86, 89, and 96. J Clin Oncol 18(4): 832-839

Schneider DT, Hilgenfeld E, Schwabe D, Behnisch W, Zoubek A, Wessalowski R, Gobel U (1999) Acute myelogenous leukemia after treatment for malignant germ cell tumors in children. J Clin Oncol 17(10): $3226-3233$

Schneider DT, Wessalowski R, Calaminus G, Pape H, Bamberg M, Engert J, Waag K, Gadner H, Gobel U (2001) Treatment of recurrent malignant sacrococcygeal germ cell tumors: analysis of 22 patients registered in the German protocols MAKEI 83/86, 89, and 96. J Clin Oncol 19(7): 1951 - 1960

Haematology and Oncology, St Anna Childrens Hospital, Vienna, Austria; S McCann Shaun McCann, Department of Haematology and Institute for Molecular Medicine St James's Hospital and University of Dublin, Trinity College, Dublin, Ireland; H Joensuu, Department of Oncology, University of Helsinki, Finland; C Mitchell, Paediatric Oncology, Oxford Radcliffe Hospital, Oxford, UK; E Plouvier, Unite d'haematologie infantile, Hopital SaintJacques, CHU, Besancon, France; R Schots, BMT Unit, Academisch Ziekenhuis-Vrije Universiteit Brussel, Brussels, Belgium; J-P Vannier, Service d'Immuno-Haemato-Oncologie Paediatrique, Centre Hospitalier Universitaire, Rouen, France; A Yalcin, Department of Haematology, Gulhane Military Medical Academy, Ankara, Turkey; MA Yesilipek, Department of Paediatric Haematology and Immunology, Akdeniz University School of Medicine, Antalya, Turkey. 